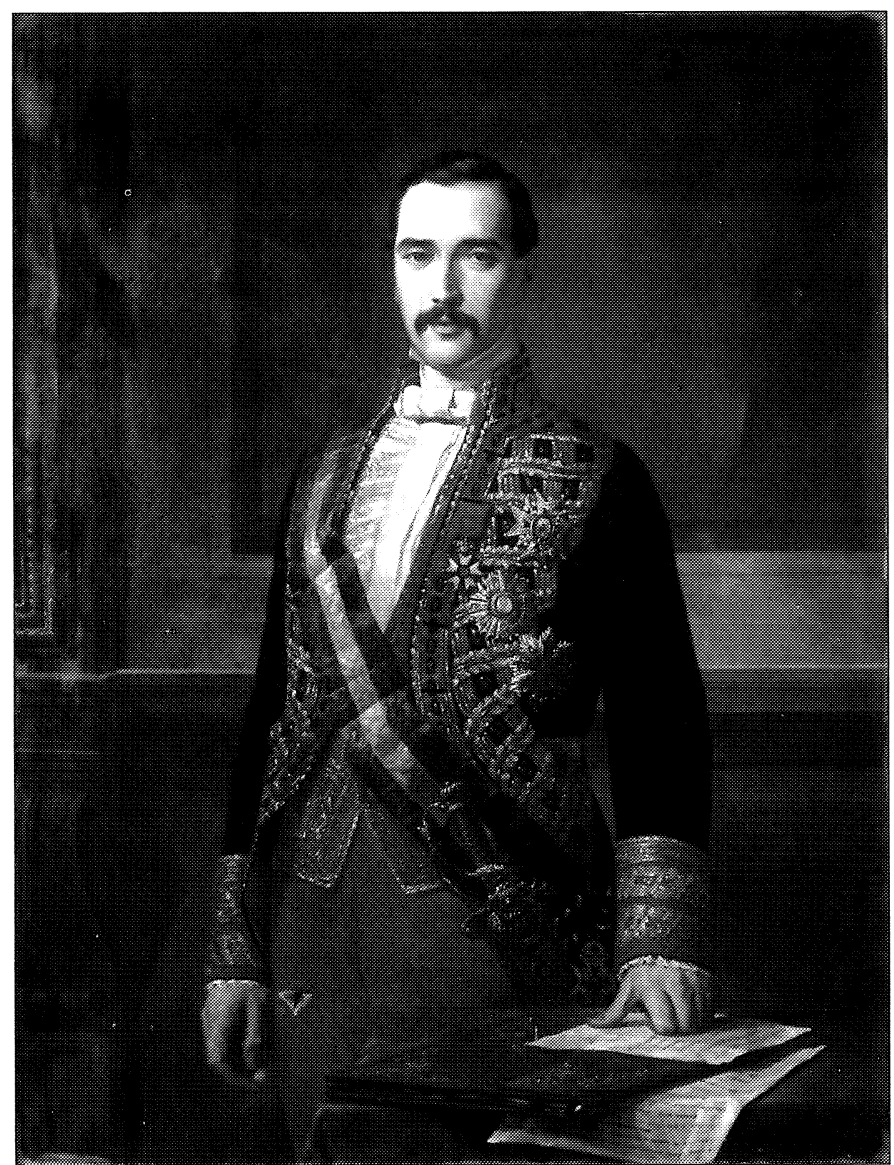

Agustín Esteban Collantes, ministro de Fomento, por Justo María de Velasco (c. 1854).

La datación del cuadro puede ajustarse a partir de la fecha de concesión de la Orden de Carlos III (1854) y el final de su mandato ministerial. Ahora bien, asalta la curiosa particularidad de que las demás condecoraciones (la Cruz de las Epidemias es anterior a la concesión de la de Carlos III), en concreto la lusa, son muy posteriores, por lo que no se hace difícil pensar que fueran añadidas con escrupuloso cuidado de la mano de Justo María de Velasco.

José Luis SÁNCHEZ GARCía

\title{
ALGUNOS DATOS SOBRE EL PALACIO ESPAÑA EN ROMA Y EL PATRONAZGO DEL CONDE DE ALTAMIRA EN SU EMBAJADA
}

En 1695 Don Luis de la Cerda y Aragón, Duque de Medinaceli, se veía obligado a abandonar su residencia en el palacio romano de la Plaza de España debido al estado de ruina causado por el desplome de gran parte del edificio.

El palacio, que nunca gozó de buena salud, fue objeto de nuevos reparos desde su adquisición para ejercer de sede de la Embajada española en tiempos del Conde de Oñate. Como que- 
dó señalado por el Conde de Altea ${ }^{1}$ aunque la compra del Palacio Monadeschi supuso un triunfo diplomático - al lograr la facción española afianzar así su dominio de la zona frente a los intereses franceses por ocupar también el viejo palacio - en realidad, la adquisición iba a suponer una sangría constante para las arcas españolas, ya que será recurrente la enfermedad crónica de este palacio, que necesitó constantes aportes de dinero para reparaciones variadas. Ya señalaba el mismo autor algunos reparos efectuados desde el momento de su adquisición en que incluso el Conde del Infantado lo habitó cuando aún no estaba completada su adecuación para alojar a tan dignos personajes. Esta va a ser la constante en la historia de este palacio. Sus sucesivos inquilinos soportarán con mayor o menor resignación los achaques del caserón, algunos llegarán a asumir su incomodidad antes que buscar otra residencia.

Incluso Don Luis de la Cerda, que lo desalojaría antes de acabar su mandato invirtió sus esfuerzos y su dinero, más concretamente el dinero ajeno, en arreglarlo, durante su embajada en Roma.

Es así que desde el principio de su estancia en la ciudad eterna, ostentando aún el título de Marqués de Cogolludo ${ }^{2}$, va a realizar numerosas peticiones de dinero para el arreglo del palacio. Sus reiteradas solicitudes se dirigen al Conde de Santisteban que ocupaba el virreinato de Nápoles. Queda claro, por estos y por posteriores documentos que aunque estaba maltrecha la situación económica del Virreinato, era la ciudad partenopea la que asumía los pagos de la Embajada Romana.

Las cartas de solicitud de dinero, dádivas y agradecimientos que se intercambian el Conde de Santisteban y el de Cogolludo, nos permiten aclarar diversas cuestiones:

El Marqués de Cogolludo se vió obligado en los tiempos de su misión no solo a realizar las que ya habían llegado a ser tareas habituales de «remiendo» del palacio, sino que además se tuvo que encargar de que se rehiciera la parte dañada por un rayo durante la misión de don Luís de Guzmán ${ }^{3}$, así para «darse principio a la composición de lo que quemó el incendio» ${ }^{4}$ acude al Virrey para que le socorra en el reparo y este muestra, aún insinuándole la estrechez de medios con que se cuentan en Nápoles, su total disposición al progreso de la obra, indicando también al enviar el dinero, su finalidad, que era para el «reparo y composición de lo que consumió el incendio en ese real palacio» ${ }^{5}$. También en estos años, para ejecutar estas obras el marqués de Cogolludo habla a su tío de un informe realizado sobre el estado del edificio, con «los medios necesarios que consideran los arquitectos como verás por la adjunta relación a fin de que se pueda tratar desde luego de la obra», que no he hallado, ya que no he encontrado la documentación que se cita en dicha carta ${ }^{6}$.

\footnotetext{
1 Conde de Altea: Historia del Palacio de España de Roma en Madrid. 1972. IMNASA. Dirección general de bienes culturales. Sobre la adquisición por Oñate del Palacio, facilitada por el papado de Inocencio X, pág 24-6; sobre el del Infantado que refleja el propósito de prestigiar la residencia de la Casa Real, pág 29; Con Castel Rodrigo etc...señala que solamente se autorizaban reformas mínimas, p. 49.

${ }^{2}$ Su nombramiento no pareció muy adecuado a la comunidad española, recibiendo numerosas críticas por su «afrancesamiento» y por su edad inexperta, como ya señala Altea, Op. Cit., y como se ve muestra por el Manifiesto contra el marqués de Cogolludo embajador de Roma (BIBLIOTECA Nacional, Manuscrito $11261 \mathrm{n} .^{\circ}$ 5): «EL EMBAXADOR DE ESPAÑA INCOGNITO/CONOCIDO/EN LA MAS NOTORIA IGNORANCIA DE SU REY/PUBLICO/EN EL MAIOR TRIUMPHO DE EL DE FRANCIA/MANIFIESTO/EN LOS MAS ENGAÑOSOS TRATADOS CONTRA EL SEÑOR EMPERADOR/EL MARQUÉS DE COGOLLUDO EN ROMA». Le sigue una carta de este al rey Carlos II indicando que todo son calumnias y que "queda prontíssimo a retirarme luego de esta ocupación y constituirme en un castillo desde donde o mis culpas me pongan en el suplicio, o mi inocencia califique la pureza de mi honor, y de mi prozedimiento, no pudiendo en forma diversa parezer en el mundo mi reputacion con una mancha tan negra porque en los vasallos»

3 Ibidem, pp. 63-64.

${ }^{4}$ Archivo Ducal de Mecinaceli (A.D.M.). Sección Archivo Histórico. Legajo 21. Ramo 1. Carta desde Roma del Marqués de Cogolludo al Conde de Santisteban, fechada en Roma el 7 de mayo de 1688, avisando del recibo de una letra.

${ }^{5}$ A.D.M. Sección Archivo Histórico. Legajo 21. Ramo 1.Carta desde Nápoles en 10 de mayo de 1688. A.D.M. Sección Archivo Histórico. Legajo 21. Carta desde Nápoles el 14 de mayo de 1688.

${ }^{6}$ A.D.M. Sección Archivo Histórico. Legajo 21. Carta desde Roma el 10 de abril de 1688.
} 
En segundo lugar, en la documentación de estos años podemos ver cómo estas tareas en las que se afanaba el Marqués, no venían motivadas tanto por el deseo de ver recobrar la dignidad al Real Palacio y reparar las partes afectadas por el fuego, como por sus ansias personales de aparentar. Don Luis, hombre destacado por su saber vivir y gusto por organizar todo tipo de fiestas para deleite del pueblo, hace referencia en todas las cartas a su prisa porque el palacio esté adecentado para el día de San Pedro. El Marqués del Carpio, en los tiempos de su misión en Roma recobró la costumbre que había existido de realizar una ceremonia que daba testimonio del vasallaje de Nápoles a la Santa Sede ${ }^{7}$, y al final de la cual, se recibía en la Embajada a los más distinguidos personajes presentes en Roma, por lo cual Don Luis de la Cerda quería que «acercándose el dia de San Pedro para el qual se necesita que esta casa esté reparada y compuesta con la decencia que es justo» ${ }^{8}$, ya que era mucha "la indecencia con que se haya esta casa y estar tan próximo el dia de San Pedro en el cualhaviendo de executar la función es necesario tener usual todas las viviendas y aún así creo que no podrá bastar por razónde la concurrencia de cortes que habrá en el quato de mi prima» ${ }^{9}$, es decir, la parte que ocupaba su mujer. A tales peticiones respondía el de Santisteban, mandándole diversas cantidades de dinero y así una de las veces adjuntando una póliza de 1.000 escudos le hace saber "que con esta cantidad aunque corta se puede dar principio a la obra y que yo quedo en el cuidado de solicitar otras para que no se zese en ella de suerte que esté cocluyda para el dia de la vigilia de San Pedro» ${ }^{10}$.

Además de las peticiones de dinero ${ }^{11}$ que permitiera acabar las obras para que el Palacio estuviese dispuesto el día de San Pedro, Medinaceli solicita a Nápoles el envío de ropa, carrozas, libreas ${ }^{12}$, todo aquello que diera mayor grandiosidad a los festejos que debía organizar, cosa que no sorprende, pues conocido es el carácter derrochador del Marqués y es lógico que quisiera en dicha fecha mostrar toda su generosidad.

Pero parte de los dineros que Don Luis gastó en los arreglos del Palacio, fueron una vana inversión, ya que antes de abandonar su misión diplomática en Roma, tuvo que asistir al derrumbamiento de un sector del Palacio. Alcolea recoge, como el ya Duque de Medinaceli ex-

7 Ceremonia descrita por el Conde de Altea. Op. cit.

8 A.D.M. Sección Archivo Histórico. Legajo 21. Carta citada del 10 abril de 1688. Roma.

9 A.D.M. Sección Archivo Histórico. Legajo 21. Carta citada del 7 de mayo de 1688. Roma.

${ }^{10}$ A.D.M. Sección Archivo Histórico. Legajo 21. Ramo 1.Carta citada del 10 de mayo de 1688. Nápoles.

$"$ Los envíos son continuados:

A.D.M. Sección Archivo Histórico. Legajo 21. Ramo 1.Carta citada del 10 de mayo de 1688. Nápoles. Adjunta póliza de 1.000 escudos.

A.D.M. Sección Archivo Histórico. Legajo 21. Carta citada del 14 de mayo de 1688. Nápoles. «En carta del 7 del corriente eres servido acusar el recibo de la letra de cambio de 952 escudos y 38 "vayoques" de moneda romana que remití el $1 .^{\circ}$ de este mes».

A.D.M. Sección Archivo Histórico. Legajo 21. Ramo 1.Carta desde Nápoles 28 de mayo de 1688. Indica que adjunta letras de 2.000 escudos.

A.D.M. Sección Archivo Histórico. Legajo 21. Ramo 1.Carta desde Roma a 4 de junio de 1688. Avisa del recibo de las dos letras, e indica «para lo cual las remitió su excelencia de cantidad de 1548 escudos moneda romana».

A.D.M. Sección Archivo Histórico. Legajo 21. Ramo 1.Carta desde Nápoles a 6 julio 1688. Acompaña letra de 4.000 escudos.

A.D.M. Sección Archivo Histórico. Legajo 21. Ramo 1.Carta desde Roma a 9 de julio de 1688. Agradece el dinero, «que le llegó muy a tiempo».

A.D.M. Sección Archivo Histórico. Legajo 21. Ramo 1.Carta desde Nápoles a 24 de agosto de 1688. Santisteban indica a su sobrino que no le puede asistir con los 7.000 ducados que le había pedido y le envía 4.000. Va con tres pólizas de 4.000 «escudos moneda de Nápoles reducidos a 38.9 y q.s 52 desta de Roma».

A.D.M. Sección Archivo Histórico. Legajo 21. Ramo 1.Carta desde Roma en 27 de agosto. Acusa y agradece el recibo de las tres pólizas.

A.D.M. Sección Archivo Histórico. Legajo 21. Ramo 1.Carta desde Nápoles en 10 de septiembre. Le indica que a pesar de la «estrechez en que se halla este real patrimonio» le remite tres letras de 4.000 ducados de esta moneda.

12 A.D.M. Sección Archivo Histórico. Legajo 21. Carta citada del 7 de Mayo de 1688. Roma. 
plica en carta del 25 de Febrero de 1695 a Santisteban, que dos días antes había caído parte del edificio que ya era inhabitable, por lo cual había solicitado informes de dicho estado, para justificar así su huida de tan ruinoso palacio. Así,los últimos meses en la ciudad eterna, los pasó don Luis en el Palacio Manfroni ${ }^{13}$ antes de pasar a ocupar su nuevo cargo en Nápoles, adonde llegó el 27 de Marzo de 1696. El joven noble dejó Roma sin la menor pena por parte de los españoles que allí residían. Pasará, desde la posición de su nuevo cargo, a ser él quien deba enviar dinero a Roma, destinado, nuevamente a las obras en el Palacio.

El sucesor en la embajada fue Luis de Moscoso y Guzmán, noveno Conde de Altamira ${ }^{14}$. A diferencia de su antecesor, parece más preocupado por terminar el palacio para que sea digna sede de la Embajada y no como medio para mostrar su ostentación.

Es de suponer, que desde su llegada a Roma, Altamira se ocupó del reparo del Palacio. Las primeras noticias que encontré de las que hacen referencia a su embajada, datan del 8 de Marzo de 1697 en que escribe al Virrey: "y así esto como la forma de ponerme aquí de asiento quedara concuydo de vuelta del viaje de Loreto, he visto el Palacio que en feneciéndose vendrá bastante comodidad pudiendo decirte con verdad es tan buena y tanta la obra que se a echo que con poca más pudiera hacerse nuevo desde los cimientos; lo que le falta es algo que se debe ya juzgo sabes lo que es aunque si no hacen una de las suyas compadeciéndome y enviando pronto socorro, parara la obra y andaré peregrinando sin cierta residencia» ${ }^{15}$. Parece pues que aún no residía Altamira en el Palacio de la plaza de España. Como era costumbre, el avance de las obras dependía de la llegada del dinero, y más en este caso, que por lo que recuerda aquí Altamira, y que ya recogía el conde de Altea, debió ser bastante importante, pues se trató de un derrumbe del techo de la escalera principal repercutiendo esta caida en la ruina de las estancias inmediatas ${ }^{16}$, y estas reformas debieron afectar al aspecto del Palacio. En Noviembre de dicho año, las obras ya debían estar bastante avanzadas, pero como indica el de Altamira en carta escrita al de Medinaceli ${ }^{17}$ el primero de este mes: «siento infinito el verme precisado a aberte de hablar de nuevo en el reparo de este Palacio, considerando lo mucho que te ha molestado su obra y lo que has exercitado tu liveralidad en los medios que has subministrado para ella; pero quando crei havia llegado el termino a concluirla, se ha reconocido que la pared principal, del costado que mira al medio dia de la Pieza de la Audiencia, amenaza ruina, ocasionada del conducto, y ventanas, que se abrieron en ella, en tiempo del Marqués del Carpio, por no haverse reparado la devilidad de sus cimientos, con unos arcos que se consideraron necesarios, y que assi mismo necesita de reparo el muro de junto a la s.ria según mas pormenor veras por las relaciones adjuntas de el cavallero Fontana, y de el Arquitecto Phelipe Marinello» ${ }^{18}$.

Aparte de hacernos una completa descripción de las reformas que había que realizar, pues Altamira se muestra muy detallista al dar todo tipo de explicaciones, da por sentado que se habían mandado importantes sumas de dinero para las obras efectuadas en el 1696-97 de las que aquí no aporto documentación, y en las que se debió llevar a cabo la reforma de la parte más importante, pero dejando una de las partes, que él considera «de las más principales» sin reformar. Lógicamente esto disgustó a Altamira, el cual se lamenta al virrey: «La mohina que esto me ha ocasionado no te sabre ponderar, pero la comprehenderas tu, conociendome, y

\footnotetext{
${ }^{13}$ Conde de Altea. Op. cit., p. 73.

${ }_{14}$ García Carrafa. Enciclopedia Heráldica y Genealógica Hispanoamericana. Vol 28, p. 182. Ocupó también los cargos de Virrey de Valencia y Cerdeña. Fallece en 1698.

15 A.D.M. Sección Archivo Historico. Legajo 2. Ramo 2. N. ${ }^{\circ} 11$

${ }^{16}$ Conde de Altea. Op. cit., p. 72.

17 A.D.M. Sección Archivo Historico. Legajo 2. Ramo 2. N. ${ }^{\circ}$ 127. Carta de Altamira a Medinaceli. 1 de noviembre de 1697.

${ }^{18}$ Documento I.
} 
considerandome en la creencia de que podia passar inmediatamente a avitar aquel quarto, de tanta mas comodidad, y gusto, que el que ahora avito, siendo bien sensible que haviendose gastado tanto en la obra, se haya dexado de reparar, una parte tän principal, como esta, en que no tiene poca culpa Phelipe, haviendo más de un año que está entendiendo en la fabrica, y no haviendo ablado hasta ahora la menor palabra en esto de la Sala de la Audiencia, y siendo precisamente indispensable el tratar de su reparo promptamente, para evitar el que todo el Salon venga al suelo, asique con gran mortificación mia, no puedo dejar de decirtelo, para que te sirvas dar la providencia que espero dever a tu fineza; y si presentemente la dificultaren la estrechez de los medios, no permitiendo dilación, la diligencia de apuntalar la pared que amenaza ruina, y de reforzar los cimientos, viniendo tu en que se haga, dispondré se empiece a trabajar». Por tanto en este tiempo se encontraba ya viviendo Altamira en el Palacio, y sabemos que Felipe Marinello, «arquitecto de su Magestad», con quién no parece estar muy conforme el de Altamira se había estado ocupando de la obra al menos desde hacia un año que es el periodo de tiempo al que alude el embajador. Desconocemos si hubo anterior participación de Carlo Fontana ${ }^{19} \mathrm{o}$ si ésta se reduce de forma puntual a este informe para esta reforma. No extraña la presencia del «arquitecto de Su Santidad», C. Fontana, ya que no había obra en Roma en la que su nombre no estuviese presente. Hombre infatigable, se conservan gran cantidad de diseños de su mano tanto de obras realizadas como de otras que no se llevaron a cabo, entre las que quizá se encuentren las realizadas para la remodelación de este edificio. Arquitecto, urbanista, ingeniero, también se conocen otros diseños destinados a ampliación de palacios que no se llegaron a realizar como el del Palacio Altieri. Tampoco es la única obra que Fontana aportó al contexto español, puesto que la obra más importante erigida en el país Vasco a finales del siglo XvII, el Santuario de Loyola, fue proyectado por él en Roma en 1681. Las indicaciones dadas por Fontana para el arreglo del Palacio se acomodan a las estrecheces que la economía del momento demandaba. «El Príncipe de la Academia de San Lucas», elegido de forma extraordinaria por segunda vez de 1692 a 1700, tuvo que ajustarse, como él mismo indica en su presupuesto, a los escasos presupuestos españoles.

El Duque de Medinaceli, que durante su embajada recurrió tan repetidamente al socorro de las arcas virreinales, ahora recibe las peticiones de Altamira, quién a diferencia de aquel no solo pide dinero, sino que informa a su sobrino de diversas noticias ocurridas en Roma ${ }^{20}$, además, por supuesto, de ponerle al día de los arreglos que se llevaban a cabo, con lo cual parece que se siguieran los informes efectuados por los arquitectos. Así le habla de los «reparos que son necesarios así en la pared de la sala de las audiencias como en el muro que va a la secretaría de este Palacio» ${ }^{21}$.

A pesar de las estrecheces económicas que al igual que sus antecesores sufrió el Conde de Altamira para reformar el siempre maltrecho Palacio, parece que dedicó más esfuerzos que su antecesor para embellecerlo. Así, en el mismo año 1697 están fechados tres recibos en los que se pagan a tres pintores por las obras realizadas para el Conde de Altamira: Bonaventura Lam-

\footnotetext{
19 Coudenhove-Erthal, E., Carlo Fontana und die Architektur des Römischen Spätbarock. Viena, 1930. WitTKower, R. Arte y Arquitectura en Italia.1600-1700. 1958. Ed. Cátedra, 1988, pp. 373-376.

20 A.D.M. Sección Archivo Histórico. Legajo 2. Ramo 2. N. 35 y 36 Informa de que el Papa estaba haciendo la obra de Nettuno, puerto donde manda a Gen.va como arquitecto. Teme que sea malo para el comercio Español ya que será refugio de corsarios y genoveses. En las N. 37 y 46 el embajador pide a Medinaceli que le diga a como está el cambio de la moneda, pues no le han hecho efectivos más ayudas de costas por no saber a cómo estaba el cambio. En la carta N. ${ }^{\circ} 42$, nuevas quejas por la realización del puerto en Anzio. En la N. 49 fechada en Roma el 14 de julio de 1697,agradece que se haya dispuesto el envío de 2000 escudos Romanos, que se entregarán a Dominico Perelli, que actuaría como agente. Este personaje aparece de nuevo en la carta N. ${ }^{\circ} 136$ y 156 y N..$^{\circ} 174$ ya que se le entregan a el 300 escudos para acudir «a los gastos de los nuevos reparos», fechado en Roma de noviembre a diciembre.

${ }^{21}$ A.D.M. Sección Archivo Histórico. Legajo 2. Ramo 2. N. ${ }^{\circ} 136$. Roma, 22 noviembre 1697. Idénticas palabras en N. ${ }^{\circ}$ 156 del 6 de diciembre.
} 
berti, Philipp Peter Roos y Gerónimo Nuzzi ${ }^{22}$. Aunque solamente en el pago a G. Nuzzi se especifique «por cuenta de las labores que hace en la obra de este real palacio», pues posiblemente fuesen obras al fresco, no cabe duda de que los cuadros realizados por los otros, aunque fuesen adquiridos con la intención de engrosar su colección particular, que desconozco, su misión inicial sería la de decorar y contribuir a embellecer las paredes del Real Palacio en Roma.

Aunque encontrar el nombre de Lamberti ${ }^{23}$ entre la documentación que hace referencia a unos pagos por obras ejecutadas para Altamira, pudiera hacer pensar en que se ocupase de la decoración de algunas salas de la Embajada, puesto que él se había ocupado ya de algunos palacios en Roma como el Palazzo Taverna (ex palacio Montegiordano para el marqués Gabbrielli), el recibo deja claro que en este caso es por el pago de dos cuadros de tema religioso. De nuevo Altamira recurre a otro artista favorecido también por el patronazgo papal como hizo en el caso de C. Fontana ${ }^{24}$. Lo curioso de este recibo del 17 de Noviembre de 1697 (fecha en la que sabemos por las cartas citadas que aún estaba vivo el Conde de Altamira) es que queda escrito por mano de Lamberti, que acusa recibo de la cantidad por pago final de los dos cuadros hechos a petición del Conde, indicando que no se pagó el de San Francisco de Borja, que fue hecho en vida del conde y que, «cuando ya había muerto, entregó él el de San Antonio», firmándolo en dicho dia.

Por lo que respecta a los cuadros encargados y pagados a Rosa da Tivoli ${ }^{25}$, seguramente pinturas de animales, se le entrega la cantidad de nuevo por medio de Dn. Luis Alvarez del Valle secretario del Conde, en fecha posterior al pago a Lamberti, dándose por sentado que el Conde estaba con vida. No es posible poder comparar las cantidades entregadas a los distintos pintores pues no se indica cuántas pinturas entregó Roos.

Sobre Geronimo Nuzzi, «pintor», desconozco otros datos. Quizá por su apellido pudiera ser pariente de Mario Nuzzi, llamado Mario dei Fiori. Debía ocuparse, por cuanto se indica en la documentación, de las pinturas al fresco en las salas del Palacio, ya que la cantidad es reducida, quizá debido a ser un pago parcial.

Esta documentación, aún siendo limitada, puede venir a aportar nuevas consideraciones en primer lugar sobre la figura del Conde de Altamira y su relación con las artes, y también sobre la labor de patronazgo de los personajes españoles, que residiendo en tierras italianas ejerciendo sus misiones diplomáticas, recurrieron a los artistas que les rodeaban, no solo para hacer acopio de sus obras y así aumentar sus colecciones particulares, sino además para embellecer los edificios, en este caso el Palacio de España, que en aquellas tierras pertenecían a la corona Española, aunque en muchos casos las reformas posteriores nos impidan conocer los resultados entonces conseguidos ${ }^{26}$.

${ }^{22}$ Documento II.

${ }^{23}$ Bonaventura Lamberti. Carpi 1652-Roma 1721.

The Dictionary of Art. Ed. J. Turner. Vol. 18, p. 320.

Thieme-Becker. Tomo XXII. pp. 255 y 256.

PASCOLI, L., Vite de pittori, scultori ed architetti moderni, scritte e dedicate (1730-36). Ed. Amsterdam 1965. Tomo II, pp. 331-339.

${ }^{24}$ RUDolph, Stella: «The "Gran Sala" in the Cancelleria Apostolica; a homage to the artistic patronage of Clement XI». Burlington Magazine. CXX. N. ${ }^{\circ}$ 906. Sept. 1978, pp. 593-601.

${ }^{25}$ Philipp Peter Roos ó Rosa da Tivoli. Lowe Rhine, 1657-Roma 1706. Desde 1691 presente en Roma.

The Dictionary of Art. Ed. J. Turner. Vol. 27, p. 135.

Thieme-Becker. Tomo XXXIII, pp. 581-582.

${ }^{26}$ Para la trasformacón de la fachada en el siglo xix: A. DoninI, «Trasformismi architettonici: la facciata del Palazzo di Spagna a Roma in una medaglia di Pio IX», in Medaglia, 2, 1973, 5, pp. 76-86. 


\section{Apéndice Documental}

\section{Documento I}

ARCHIVO DUCAL DE MEDINACELI (A.D.M.) Sección Archivo Historico. Legajo 2. Ramo 2.

\section{N. ${ }^{\circ}$ 128. Relación de Carlo C. Fontana}

Havendomi imposto l'ecce.mo signore Conte d'Altamira Ambasciatore della Real Corona Cattolica in Roma di riconoscere l'apparenza di alcuni peli conticui al cornicione dorato nella salla d'Udienza verso strad tratina; onde data le impositione si è indagato da me sottoscritto assieme non il signor Filippo Marinello Ingegniere, et Architetto di sua Magestà la causa, et ysaminato, e riconosciuto la qualita del muro ove risiedono d.i (detti) peli, e si è indagato totalmente la d.a (deta) pariete circa la sua qualità di muro che la compongono; come anco l'ufficio con cui debe operare de.a (detta facciata.

$\mathrm{Si}$ è trovato la d.a muraglia di tufi del suo originari antico stato alquanto macarata(mascarata?) e scompaginata e verso anco inhabilitato da 1 forami, che in detto muro si trovano di varie porte e finestre con un condetto tagliato in esso, che sviscerano? La collocatione del detto muro e si è trovato il fondamento che lo regge di profondità di palmi dodici in quindici in circa cosi riferto da Mastro dominico ragazzone CapoMaestro sopra il qua fondamento si vede riattato nelle parti vicine al terreno del cortile con una fodara di muro ben compaginata, il qual muro si restringe in una piccol. Parte e non nella totale estensione dove provengono 1 difetti, et appariscono le crepature, le q.li (quali) feriscono? A corrispondenza del pelo sopra il prenominato cornicione dalla sala, et havendo pienamente fatto la sue dovre rifflessioni assieme ed il igr. Filippo sud.o, e capo Maestro Stante la relassatione della propria materia $\mathrm{p}$ esser di tufi al quanto disciolta si è stabilito secondo la nostra peritia e prattica di porre ir rimedio aeciò no sia: no preforati 1 muri, in modo che la sala, et altro non venga impedita per La propria habitatione presente di S.E.E di rinforzare con tré pilastri, cioè due nei lati della pariete sopranominata, l'altro nell' angolo della rivolta che costituisce il Gabinetto conticuo li quali pilastri havenvanno di proiettura circa palmi quattro fora della linea del muro con altri palmi due da entrarsi nelle viscere de 1 proprij muri di una longitudine suficiente,con suoi fondamenti di rinforzo bisognando sotto le pariette dove appariescono 1 danni, q.li pilastri alzari a ppendicolo sino al piano nobile scrivano y. Una firme base, e sostegno valido a vaggere con un volto di muro la loggia triangolare di legno, la gravezza della quale opera ad inhabilitare la d.a muraglia inhabili, e dal piano nobile in su' caminare ed 1 medemi pilastri sino al tetto; ma di meno proiettura per po poter fra li medemi voltar gl'archi per lasciare illeso li forami delle finestre, a qsta nostra peritia, e modo sarà la minor spesa per non entrare a svegliare con muraglie totali la quiere? Del Palazzo in quella parte, et essendo evidenti 1 bisogni che non ammettono la procastrinatione del rimedio si in questa parte; come anco in una piccol parte sotto le ringhiere verso la piazza maestra sotto la stanza della secretaria, come nell'ordinario venturo S.E. Signor Ambasciadore haverá un altra simil notitia ed 1 disegni dimostrativi delle v.ni donde vien fondata la nostra relatione, e peritia, nelli quali vi sarano anco li scandagli delle spese che perciò p. L'ossequio, che si debe ho sotto scritto la presente di mia propria mano. Questo? 31 setembre di 1697.

Carlo Car. Fontana. Arch. Di sua Santità

N. ${ }^{\circ}$ 129. Relación de los gastos, firmada por Filippo Maniello, pero la letra es la misma que la del n. ${ }^{\circ} 128$, que parece ser la misma de la firma de Fontana.

Havendomi V.E. comandato chi io facci il scandaglio della spesa che anderà a far 1 pilastri con loro fondame.ti: passonati e il tutto come, si descrive nela relatione fatta dal Cavalier Fontana Architetto di S. ${ }^{a}$ s.ta me covnito?; per il riparo del muro della sala d'Udienza, che stà conticuo al Gabinetto dove proprio è la liggietta in triangolo e questi pilastri tutti co.pti di Colla, imbiancati, et incluso ci la pontellatura del muro vecchio seandargliato importano scudi cinque cento novanta sette 597

Per il riparo del muro sotto la secretaria, qu.le debe appedarsi da dentro la cantia, e far alcuni pilastri di mattoni, quale riparo si nomina anco nella sud ${ }^{\text {a }}$ relatione che scandagliate importano scudi novanta sei 96

Che uniti a qelli soprad.i impo.rno 693

Y questo è quanto l'e.s. mi ha comandato e que..o che possi riferire restando..

Roma p.mo nbre 1697.

Documento II

BIBLIOTECA NACIONAL. Manuscrito 18643

N. ${ }^{\circ} 51$

«Recibo de 63 escudos por precio de los cuadros de S. Francisco de Borja y S. Antonio de Padua que el pintor Buenaventura Lambert hizo para el Conde de Altamira [Roma] 17 Noviembre de 1697».

Io infraschitto o ricevutto del SS.mo sig.. ${ }^{\circ} \mathrm{D}$. Luiggi Alvarez del Valle scudi sesantatre monetta qualli sonno per prezo e finali pagamen.to dei quadri di San Francesco Borgia e del Sn Antonio di Padua fatti per ordine di sua ecc.a il sig.r Conte 
di altamira Felice memoria. Non avendo mai pagatto quello di S. Francesco fatto in vita di sua ecc.ha, e doppo morto li o consegnatto il san Antonio et in Fede questo de 17 Novembre 1697.

Io Bonaventura Lamberti mpp.(firma autógrafa)

Bentura Lambert

N. 229

«R.vo de 63 escudos por precio del quadro de s.n Francisco dde Borja que havia pintado para el Conde mi S.r y entregado viviendo S.E. y del de Sn Antonio de Padua que consiguió después de su muerte.»

Pass.do.

N. ${ }^{\circ} 52$

Libranza de 70 escudos hechas por el Conde de Altamira a favor del pintor Phelipe rosa por cuenta de obras y recibí autógrafo firmado por el mismo pintor. Roma 10 de Diciembre de 1697.

Dn. Luis Alvarez del Valle mi S.rio de Camara a cuia q.ta corre p.r haora la cobranza y distribucion de mis sueldos y rentas, dò y entregue a Phelipe Rosa Pintor= Setenta escudos deesta moneda por q.ta de las pinturas que me esta haziendo; que en virtud deesta libranza y su recibo haviendose tomado la razon en mi contaduria, se le haran buenos. Roma 10 de Diz.re de 1697.

Al conde de altamira

Tomó la razón en los libros de la contaduris de S.E A P.. ${ }^{19}$. Joseph Borg.s de Contreras. (firma autógrafa).

V.E. libra a Ph.e Rosa Pintor= 070 es. Por. Qu.ta de las pinturas que está haz.do.

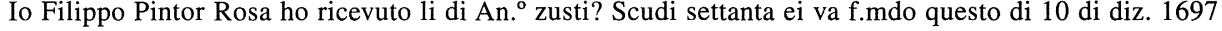

Io Fillippo Pin.t. Rosa mano propria.(firma autógrafa)

N. 54

Libranza de 5 escudos hecha por el Conde de altamira a favor del pintor Gerónimo Nuzzi por cuenta de obras. Roma 11 de Diciembre de 1697 (Firma del pintor).

D. Luis Alvarez del Valle mi secretario de Camara a cuya quenta corre por ahora la cobranza y distribucion de mis sueldos y rentas y entregue a Geronimo Nuzzi Pintor cinco escudos desta moneda, por quenta de las labores que haze en la obra deste Real Palacio, que en virtud de esta libranza y su recibo haviendose tomado la razón en mi contaduria se le harán buenos. Roma 11 de x.re de 1697.

Al Conde de Altamira.

Tomé la razón en los libros de la contaduria de S.E a p.. 20 .Joseph Borg.s de Contreras(firmado)

-V.E. libra a Ger.mo Nuzzi pintor 5 esc.os por q.ta de las labores que haze en la obra de Palacio.

María Jesús MuÑoz GonZÁLez

\section{FRANCISCO RIZI «RESTAURADOR»DE PINTURAS}

Una faceta hasta ahora poco conocida de Francisco Rizi de Guevara, pintor de cámara de su Majestad y ayuda de la Furriera ${ }^{1}$, es su labor de «restaurador» de pinturas. Sin embargo, tenemos constancia de que en el tiempo que desempeñó el cargo de pintor de cámara de don Juan José de Austria (del 17 de enero de 1678 al 17 de septiembre de 1679) ${ }^{2}$, tuvo que acometer, entre otras funciones, el trabajo de «Pintar y renovar» algunos cuadros de la colección de Su Alteza.

\footnotetext{
1 Sobre su papel de pintor ver bibliografía más reciente: Pérez SÁnChez, A. E.: Carreño, Rizi y Herrera y la pintura madrileña 1650-1700, Madrid, 1986. Agulló y Сово, Mercedes: «Antonio y Francisco Rizi», Anales del Instituto de Estudios madrileños, 1996, pp. 75-97. Entre las últimas aportaciones figuran: Ruiz GómEz, Leticia: «Dos nuevos lienzos de escultura madrileña en las Descalzas Reales de Madrid, y una hipótesis sobre la devoción al Santo Sepulcro», Reales Sitios, n. ${ }^{\circ} 138$, 1998, pp. 55-63. GutIÉRrez PAstor, Ismael: «Un proyecto de Francisco Rizi para la cúpula de San Antonio de los portugueses», A.E.A. 1999, pp. 531-535.

${ }^{2}$ González Asenjo, Elvira: «Artífices y tasadores de la Capilla de Nuestra Señora de la Concepción, más conocida como capilla del Milagro de las Descalzas reales (1678)», A.E.A., 1999, p. 584, nota 8.
} 